\title{
LOS POSGRADOS DE COMUNICACIÓNY SUS APUESTAS SOCIALES DE CONTENIDO. ¿SE ESTÁN RESPONDIENDO A LAS DEMANDAS EN EL MARCO DEL ESPACIO EUROPEO DE EDUCACIÓN SUPERIOR (EEES)?
}

Silvia Olmedo-Salar1: Universidad de Málaga. España silviaolmedo@uma.es

Isabel Ruiz-Mora: Universidad de Málaga. España isabelruiz@uma.es

\section{RESUMEN}

La adaptación al Espacio Europeo de Educación Superior (EEES) ha brindado al conjunto de las Universidades españolas la oportunidad de incorporar a sus programas académicos nuevos ejes de contenidos que fortalezcan y promuevan demandas que la sociedad va requiriendo dada la propia dinámica de las estructuras políticas, sociales, culturales y económicas. En este sentido, si partimos de la premisa que la comunicación tiene un valor crucial como dinamizador y componente imprescindible en la actual Sociedad del Conocimiento, las facultades que abarcan dicha disciplina deberían responder en sus nuevos programas docentes a las tendencias que la sociedad solicita en su camino. Por ello, en este trabajo pretendemos identificar si este proceso se ha acometido en dos ámbitos que en los últimos años están acaparando enfoques de interés social dadas sus implicaciones de impacto social, medioambiental, político y económico como son la Cooperación Internacional para el desarrollo y la Responsabilidad Social y en concreto, en los posgrados ofertados en el conjunto de las Facultades de Ciencias de la Comunicación.

PALABRAS CLAVE: Comunicación - Enseñanza - Responsabilidad Social Posgrados

\footnotetext{
1 Autor correspondiente

Silvia Olmedo-Salar: Departamento de Periodismo. Facultad de Ciencias de la Comunicación. Málaga. España

Correo: $\underline{\text { silviaolmedo@uma.es }}$
} 


\title{
THE GRADUATE OF SOCIAL COMMUNICATION AND ODDS OF CONTENTS. ARE ANSWERING THE DEMANDS UNDER THE EUROPEAN HIGHER EDUCATION AREA (EHEA)?
}

\begin{abstract}
Adaptation to the European Higher Education Area (EHEA) has provided to all Spanish Universities the opportunity to incorporate their new areas of academic programs to strengthen and promote content that society demands will require given the dynamics of the political, social, cultural and economic. In this sense, if we assume that communication is a critical value as a dynamic and essential component in today's knowledge society, the faculties that cover the discipline should respond in their new curricula to trends in society asks way. Therefore, in this work we try to identify if this process has been undertaken in these two areas, that in recent years are attracting public interest approaches for its implications for social, environmental, political and economic such as the International Cooperation for Development and Social Responsibility and in particular, graduate programs offered in all the Faculties of Communication Sciences.
\end{abstract}

KEY WORDS: Communication - Education - Social Responsibility - graduates

\section{INTRODUCCIÓN}

\subsection{Nuevas circunstancias, nuevas realidades en la Educación Superior}

La configuración del marco estructural universitario que ha conllevado el ajuste para la adaptación al Espacio Europeo de Educación Superior (EEES) se ha culminado en España en el curso 2010/2011, año que ha supuesto congratularse del intenso esfuerzo iniciado en el 2006, aunque todavía queda mucho por perfilar, propio de una reestructuración de tal magnitud, que ha supuesto un vuelco a los esquemas tradicionales de la hasta ahora idiosincrasia universitaria española.

En el camino recorrido, el mérito de la tensión de la partida jugada está repartida y distribuida en el conjunto de instituciones universitarias, públicas y privadas, la Administración Pública, los/las docentes, en el conjunto del alumnado y el personal administrativo y de servicio (PAS), cuyos esfuerzos han sido notorios para la consecución del logro de una normalidad del estamento universitario en un tránsito nada fácil. 
El Real Decreto (RD) 1393/2007, que regula y establece el marco legal de la convergencia hacia el EEES del sistema universitario español, ha sido la guía que ha marcado las pautas del proceso, estableciendo los tres ciclos universitarios pertinentes: grado (primer ciclo), máster (segundo ciclo) y doctorado (tercer ciclo).

En este trabajo nos centraremos en los dos estadios últimos, que están recogidos en el RD en los artículos 10 y 11, alcanzando su protagonismo en los capítulos IV y V. Debemos precisar que el RD en su conjunto está distribuido en seis Capítulos, once disposiciones adicionales, cinco transitorias, una disposición derogatoria única y cuatro disposiciones finales.

En los artículos mencionados de másteres y doctorados se incide en la especialización de los egresados con la introducción del EEES a través de los másteres de carácter especializado o multidisciplinar mientras que en el doctorado los estudiantes tienen como fin la formación avanzada en las técnicas de investigación.

En los másteres se determinan tres tipologías: el profesional, aquellos destinados al fortalecimiento de competencias y habilidades profesionales, el investigador, que tiene como finalidad la capacitación investigadora, y el académico, que aúna los dos anteriores.

Ya en los capítulos se adentra en las particularidades estructurales de los másteres y doctorados verificados, que les otorga el apellido de "oficial", estableciendo una distinción con aquellos otros programas que no tiene tal entidad. La carga de créditos conocidos como los ECTS (European Credit Transfer System) en los másteres oscila entre los 60 y los 120 ECTS, dependiendo de la duración de los cursos, que pueden ser de uno o dos años. Para cubrir los ECTS, se contemplan materias obligatorias, optativas, prácticas, trabajos dirigidos o aquellas actividades pertinentes según el máster y la presentación del trabajo Fin de Máster, que puede variar de los 6 a los 30 ECTS. Para poder optar al doctorado, necesariamente debe haberse realizado y superado un máster oficial, y la conclusión del doctorado tendrá como culminación la tesis doctoral.

La adaptación al EEES ha supuesto que todavía convivan los másteres oficiales con aquellos expedidos por las propias universidades, si bien la diferencia entre unos y otros es notoria. Como señalan $\mathrm{M}^{\mathrm{a}}$ Teresa García y Mónica Viñarás (2010, p.95), los máster expedidos por cada universidad son los denominados "Magíster" y no cuentan con el reconocimiento oficial de las administraciones competentes en la enseñanza universitaria superior frente a los másteres oficiales que tienen validez en todos los estados miembros de la Unión Europea, favoreciendo la movilidad de los egresados, además por qué no decirlo, facilitando los trámites burocráticos de reconocimiento de la especialización. 
Por lo que los másteres oficiales cuentan con el reconocimiento académico ministerial que lo convierte en un título reglado, otorgándole un rango que lo sitúa en una posición preferente en comparación con los "Magíster". Otra de las características distintivas es el precio pues los másteres oficiales tienen precios públicos por lo que están regidos y estipulados por la Administración, a diferencia de los títulos propios cuyos precios son libres y fijados de forma independiente por cada una de las universidades.

\section{2 ¿Cómo ha respondido la "arquitectura académica" española de Posgrados ante el EEES?}

En el curso 2010/2011, el sistema universitario español ha experimentado una plena adaptación al EEES y, además de la oferta de 2.338 grados, se han ofrecido 2.429 másteres y 1.624 doctorados verificados (Ministerio de Educación, 2011).

Desde la implantación oficial de los másteres oficiales en el curso 2006/2007, se ha detectado un incremento notable y destacable en la oferta de posgrados que las universidades españolas se han aventurado a ofrecer. De hecho, los programas de másteres casi se han cuadriplicado del curso 2006/2007 al pasado curso 2010/11, pasando de los 676 a los 2.429 mencionados. Según los últimos datos recogidos pertenecientes al curso 2009/2010, además de la incremento del número de titulaciones de máster, el alumnado ha ido creciendo, registrándose una clara tendencia en los últimos años en la conclusión de los estudios de posgrado.

Si en el curso 2006/2007 sólo concluyeron el 23,5 \% de los alumnos/as matriculados casi se duplica en el curso 2009/2010, que alcanzó el 43,4\%, manteniéndose en 36,6\% y $35,1 \%$ en los cursos 2007/2008 y 2008/2009, respectivamente.

Además, se ha pasado de 16.636 estudiantes de másteres en el curso 2006/2007 a 81.840 estudiantes en el curso 2009/2010, es decir, se han multiplicado por cinco el grueso del alumnado (Ministerio de Educación, 2011).

Es preciso tener claro que la salida profesional de los universitarios se constituye como uno de los objetivos fundamentales de las universidades en el EEES, meta "que pasa casi inevitablemente por la especialización a través de las enseñanzas de postgrado" (García, Viñarás, 2010, p.89), por lo que podemos señalar que la obtención de un máster oficial es un incentivo para que el alumnado vea reforzada sus expectativas profesionales, constatando los beneficios de garantía que supone a nivel europeo tal reconocimiento. 


\subsection{Comunicación y posgrados: puertas abiertas a la oportunidad en el EEES}

Consideramos que no existen dudas en el hecho que el nuevo marco académico diseñado a raíz de la introducción del EEES y el escenario, que se ha configurado con las pautas en los posgrados, se pretende responder a las demandas de la sociedad, dando cabida a las tendencias sociales, económicas, culturales y tecnológicas, entre otras.

En este panorama, la comunicación y la información son baluartes indispensables en la actual Sociedad del Conocimiento, por lo que se plantea un momento interesante y de oportunidad en la oferta de másteres y doctorados. Son numerosos los factores que están contribuyendo al fortalecimiento de escenarios comunicacionales, que las Universidades pretenden suplir como son los nuevos marcos comunicativos fruto de la aplicación de las nuevas tecnologías, especialmente en el campo de Internet, y la expansión de un potente mercado audiovisual, que no para de abrir nuevas vertientes de negocio. Además, como apunta Miguel Ortiz Sobrino (Ortiz Sobrino, 2009, p. 39):

La aparición de nuevas operadoras en sistema de TDT, la emergente televisión de movilidad, la IP televisión, los medios de comunicación interactivos y la aparición de nuevos formatos audiovisuales y multimedia, son otros factores que también están propiciando el desarrollo económico y el entramado empresarial del mercado de los "media" en España [...]

Esta coyuntura impulsa y promueve una atmósfera favorable para que los egresados, que encuentran dificultades para la inserción laboral, opten por una mayor especialización con el objetivo de poder competir con mayores conocimientos en el mercado laboral. Además, el nuevo modelo educativo basada en la adquisición de competencias, entendida como la capacidad de la aplicación de los conocimientos, destrezas y habilidades que los futuros profesionales deben poseer y poner en práctica (Flores, Roca \& Tena , 2010, p. 54), se presenta como un buen revulsivo para motivar un mayor interés en los procesos de aprendizaje.

Sin embargo, lo interesante de la propuesta es unir la vertiente de la comunicación con incipientes derroteros que se van apuntalando en el tejido social y económico y que desde el frente de la comunicación deben ser tenidos en cuenta para ir creando soportes que impulsen su estudio y su capacitación. En este camino, se aventura la senda de la Cooperación Internacional para el desarrollo y la Responsabilidad Social desde la perspectiva comunicacional, ámbitos que abordamos en este trabajo.

\subsection{Cooperación Internacional para el Desarrollo y Responsabilidad Social: ¿Invitadas?}


Si la aplicación del EEES ha supuesto la posibilidad de aventurarnos a nuevos formatos metodológicos, de aprendizaje por competencias, de funcionamiento de trabajo con una evaluación continua, al impulso de dinámicas colaborativas o al favorecimiento de la movilidad de la comunidad universitaria, ejes marcados en el Real Decreto 1393/2007, es recomendable y ciertamente necesario, el incidir y recoger en la oferta académica la premisa que señala la normativa y que indicamos:

Finalmente, se debe tener en cuenta que la formación en cualquier actividad profesional debe contribuir al conocimiento y desarrollo de los Derechos Humanos, los principios democráticos, los principios de igualdad entre mujeres y hombres, de solidaridad, de protección medioambiental, de accesibilidad universal y diseño para todos y de fomento de la cultura de la paz" (BOE, 2007)2

Es en esta indicación donde cobran especial relevancia los ámbitos propuestos en nuestra investigación como son la Cooperación Internacional para el desarrollo (CID) y la Responsabilidad Social. Los valores representados por ambos campos de trabajo se adecúan de forma pertinente a los principios propugnados, debiendo ser defendidos por las instituciones académicas.

Por un lado, la CID lleva aparejada un grueso de imaginarios como la solidaridad, los derechos humanos y la justicia social y dada su propia esencia, constituye un aspectos clave en la acción de los Estados democráticos respondiendo "al mandato contenido en el preámbulo de la Constitución española de colaborar en el fortalecimiento de unas relaciones pacíficas y de eficaz colaboración entre los pueblos de la Tierra" (Ministerio de Asuntos Exteriores y Cooperación, 2009, p.14). Por otro, la Responsabilidad Social contiene una serie de compromisos a los que la Universidad "per se" debe contribuir, entendiendo como Responsabilidad Social, aquella:

responsabilidad de una organización ante los impactos que sus decisiones y actividades ocasionan en la sociedad y el medio ambiente, mediante un comportamiento ético y transparente que: contribuya al desarrollo sostenible, incluyendo la salud y el bienestar de la sociedad; tome en consideración las expectativas de sus partes interesadas; cumpla con la legislación aplicable y sea coherente con la normativa internacional de comportamiento; y esté integrada en toda la organización y se lleve a la práctica en sus relaciones (CapacitaRSE, 2010).

\footnotetext{
2 Real Decreto 1393/2007, de 29 de octubre, BOE. 30 Oct. 07. Disponible en: http://www.boe.es/boe/dias/2007/10/30/pdfs/ A44037-44048.pdf.
} 
Por las bases en las que se consolidan la CID y la RS consideramos que la Universidad como institución de generación de conocimientos y ente, cuya simiente tiene un carácter de compromiso social, debe asumir e incentivar su inserción en la oferta académica. Su introducción, no sólo debe responder a principios de lógica que sería lo aconsejable- sino reforzada por las indicaciones fijadas por el EEES, pudiendo ser además una seña de identidad al otorgar un valor añadido en el escenario universitario y de cara al futuro de los egresados como ciudadanos comprometidos, críticos y actores de nuevas dinámicas sociales con principios éticos y democráticos. Estos principios deben ser tenidos en cuenta si desde el propio EEES se aboca a la educación permanente, que había sido bandera de diferentes teóricos e investigadores:

Ya el francés Claparede había escrito: ‘La educación es vida y no preparación para la vida'. José Martí predicó que el ser humano se educa de la cuna a la tumba. Y Pablo Freire sostiene que 'el ser humano es un sujeto educable mientras vive' . (Tünnermann, 1998)

Al determinarse la realización de los máster y doctorados como el camino de la especialización de los egresados, entendemos que conlleva aparejado un proceso de maduración del alumnado, consideramos que la inclusión de dichos ámbitos en los citados ciclos formativos serían pertinente y más cuando hablamos de la disciplina de la Comunicación, que rige la Sociedad del Conocimiento en la que nos encontramos inmersos.

\section{METODOLOGÍA}

Por las premisas dispuestas, este trabajo pretende dar respuesta a la inquietud de conocer si el escenario de los posgrados en el ámbito de la Comunicación en su adaptación al EEES está considerando la introducción de materias y programas que atiendan las demandas en el campo de la CID y la Responsabilidad Social. Entendemos que son ámbitos que están alcanzando un pulso social que debe ser considerado desde el sector académico y que deben ser integrados con decisión desde la perspectiva comunicacional, al promover valores enraizados en principios democráticos y de justicia social. Por ello, seaboga para que se contemple una planificación en comunicación que promulgue e inste a compromisos con sus entornos de proximidad, que contengan los principios que representan la Cooperación Internacional para el desarrollo y la Responsabilidad Social.

Si bien, los objetivos específicos que nos hemos marcado para esta investigación son:

a) Identificar los programas de posgrados en comunicación en el campo de 
b) Conocer si la adaptación al EEES ha incidido en la introducción de la Cooperación Internacional y la Responsabilidad Social en los posgrados de comunicación.

c) Determinar las principales vinculaciones y nexos de estudio en los posgrados de comunicación relacionados con la Cooperación Internacional para el desarrollo y la Responsabilidad Social.

Para lograr dar respuesta a estos objetivos, se han seleccionado aquellos programas de máster y doctorado oficiales de diez facultades de Ciencias de la Comunicación españolas. La metodología que aplicamos consiste en un análisis de contenido, de carácter cuantitativo y cualitativo, de los estudios de posgrado que componen la muestra objeto de estudio. Esta técnica nos permite contrastar la relación existente entre diferentes variables y la comparación de los contenidos que aparecen en las webs analizadas (Igartua, 2006), facilitando la obtención de resultados y posteriores conclusiones.

El corpus de nuestra investigación está compuesto por el conjunto de facultades de Comunicación seleccionadas, cuya elección se ha regido por dos principios, independientemente de la concesión pública o privada de las mismas:

a) -Antigüedad de la institución académica

b) -Oferta académica de la facultad en los estudios de las tres ramas del conocimiento: Periodismo, Relaciones Públicas y Publicidad y Comunicación Audiovisual.

El acceso al contenido se ha materializado a través de los sites corporativas de las facultades de Comunicación de las universidades que componen nuestra muestra, recogida en el siguiente cuadro, que ilustra de forma concisa la selección tomada: 
Tabla 1. Muestra

\begin{tabular}{|c|c|}
\hline Universidad & Web de acceso \\
\hline $\begin{array}{l}\text { Facultad de Ciencias de la } \\
\text { Información (Universidad } \\
\text { Complutense Madrid,1971) }\end{array}$ & www.ucm.es/centros/webs/fcinf/ \\
\hline $\begin{array}{l}\text { Facultad de Ciencias de la } \\
\text { Comunicación (Universidad } \\
\text { Autónoma de Barcelona, 1971) }\end{array}$ & www.uab.es/comunicacio/ \\
\hline $\begin{array}{l}\text { Facultad de Ciencias de la } \\
\text { Comunicación (Universidad de } \\
\text { Navarra, 1971) }\end{array}$ & www.unav.es/fcom \\
\hline $\begin{array}{l}\text { Facultad de Humanidades y } \\
\text { Ciencias de la Comunicación (CEU } \\
\text { San Pablo, 1975) }\end{array}$ & www.humanidades.uspceu.es \\
\hline $\begin{array}{l}\text { Facultad de Ciencias Sociales y de } \\
\text { la Comunicación, (Universidad de } \\
\text { El País Vasco, 1981) }\end{array}$ & $\begin{array}{l}\text { www.gizarte-komunikazio- } \\
\text { zientziak.ehu.es }\end{array}$ \\
\hline $\begin{array}{l}\text { Facultad de Comunicación } \\
\text { (Universidad Pontificia de } \\
\text { Salamanca, 1988) }\end{array}$ & www.comunicacion.upsa.es \\
\hline $\begin{array}{l}\text { Facultad de Ciencias de la } \\
\text { Comunicación (Universidad de } \\
\text { Sevilla,1989) }\end{array}$ & Www.fcom.us.es \\
\hline
\end{tabular}




\begin{tabular}{|l|l|}
\hline Facultad de Ciencias de la & www.cccom.uma.es \\
Málaga, 1992) & \\
\hline Facultad de Comunicación & www.upf.edu/facom/es \\
( Universidad Pompeu Fabra,1993) & \\
\hline Facultad de Ciencias de la & www.blanquerna.url.edu \\
Comunicación (Universidad & \\
Ramón Llul, 2003) & \\
\hline
\end{tabular}

De acuerdo a la técnica seleccionada y a los objetivos planteados, hemos diseñado una ficha de análisis que se ha aplicado a las distintas web de las facultades. Esta ficha se compone de dos apartados principales sobre los que se estructura los diferentes descriptores que guiarán el análisis:

La primera parte, se centra en los máster, cursos de experto y doctorados ofertados por cada una de las facultades de la muestra recogiendo aquellos datos que hemos considerado oportunos para el análisis y adecuando los descriptores al ámbito investigado, CID y Responsabilidad Social:

\section{a) Identificación}

(Universidad, título, créditos ECTS, duración, interuniversitario, internacional, precio, antigüedad, número de estudiantes admitidos).

b) Descriptores de temáticas principales que se indican en el título de la asignatura o en la denominación del título académico o en los asuntos que se tratan en ella. Cooperación Internacional (Derechos Humanos, Cultura de Paz, Género y Desarrollo, Interculturalidad, Desigualdad/Sensibilización, Cooperación Internacional para el desarrollo). Responsabilidad Social (calidad y normativas, formación/educación, sostenibilidad/medio ambiente, gestión de la RS, imagen y reputación, políticas e iniciativas RS, issues management, públicos/stakeholders, Responsabilidad Social Universitaria (RSU), ética y deontología).

c) Vínculos con la comunicación, aspectos comunicaciones que aparecen asociados al título académico o la materia. Cooperación Internacional 
organizacional, relación con los medios, comunicación para el desarrollo, otros). Responsabilidad Social (Gabinetes de comunicación, comunicación estratégica, comunicación social, Relaciones Públicas, profesionalización),

d) Especificaciones: cuáles son las líneas (investigación, profesional), si se especifican objetivos y competencias, perfiles (alumnado y docentes) acuerdos con empresas, fundaciones, ONG.

La segunda parte, se ha estructurado para canalizar las asignaturas que pudieran ser ofertas dentro de másteres y doctorados que tratasen ambos campos de conocimiento. A los datos de la identificación, de los descriptores temáticos, vinculación con la comunicación, se le ha añadido las categorías de optativa o troncal, la cantidad de créditos que representan dentro del programa de máster/doctorado y si es teórica, práctica o práctica-teórica.

\section{ANÁLISIS Y DISCUSIÓN}

Las muestras analizadas nos arroja el siguiente escenario:

a) De las diez facultades del ámbito de la Comunicación, ninguna ofrece un máster oficial íntegro, verificado por la ANECA, que vincule y se centre en el campo de la Cooperación Internacional para el desarrollo o la Responsabilidad Social y la disciplina de la comunicación, únicamente quedando rescoldos para ello en módulos de especialidad o en asignaturas integradas en algunos programas de máster.

b) La presencia más significativa en el campo vinculado al desarrollo y la comunicación, que enlaza con la apuesta de la CID, se contempla en la Facultad de Ciencias de la Información de la Universidad Complutense, que ofrece el módulo de especialidad "Comunicación, cambio social y desarrollo", que aglutina un total de 48 ECTS, y es optativo. Por su parte, el Máster en Investigación en Comunicación y Periodismo de la Facultad de Comunicación de la Universidad Autónoma de Barcelona ofrece el módulo de especialidad "Comunicación y la cooperación para el desarrollo", que contempla 80 horas presenciales con un valor de 10 ECTS.

c) En lo que respecto a las asignaturas impartidas, el Máster en Comunicación y Cultura de la Facultad de Ciencias de la Comunicación de Sevilla ofrece la asignatura "Mediación e Interculturalidad". Por su parte, el Máster en Gestión Estratégica e Innovación en Comunicación de la Facultad de Ciencias de la Comunicación de la Universidad de Málaga contempla la asignatura "Comunicación solidaria. La comunicación en las organizaciones sociales y el Tercer Sector" mientras que el Máster en Investigación en Comunicación Periodística, de la misma institución, ofrece la asignatura "Comunicación, cambio social y desarrollo. Los nuevos imaginarios de la información participativa". 
De las asignaturas indicadas, la primera es troncal y las restantes optativas, validándose por 3 y 4 ECTS. En dos de las tres asignaturas indicadas se recogen sus objetivos y competencias, y el profesorado académico es el eje vertebrador de las asignaturas, detectándose la intervención de un único profesional.

d) En temáticas vinculadas por las asignaturas y contenidas en los programas, la interculturalidad, la comunicación para el desarrollo/comunicación solidaria, género y desarrollo, y cambio social son las líneas prioritarias.

e) En la oferta académica de posgrados de la muestra seleccionada, únicamente hay una materia relacionada con la Responsabilidad Social, perteneciente al Máster en Comunicación Corporativa de la Facultad de Ciencias Humanidades y Comunicación CEU San Pablo titulada "Responsabilidad Social Corporativa y desarrollo sostenible", con el valor de 1 crédito. En el contenido de la misma, cobran relevancia los descriptores temáticos relacionados con la imagen corporativa, issues management, sostenibilidad y medio ambiente, vinculado con comunicación estratégica y la comunicación empresarial.

f) Respecto a los doctorados, se vislumbra un camino aún escasamente definido en sus planificaciones. De hecho, en algunas facultades se indican los doctorados identificándose las líneas generalistas de los mismos o escuetos apuntes de las líneas de investigación pertinentes, mientras que en otras no aparece.

g) En la Facultad de Ciencias de la Información de la Universidad Complutense de Madrid, una de las líneas de investigación correspondiente al programa "Gestión de la Comunicación, Comunicología" abre la vertiente para la Responsabilidad Social, cuyo cupo asciende a 35 plazas.

h) Por su parte, la Facultad de la Universidad Ramón Llull únicamente se especifica un doctorado genérico denominado "Doctorado en Estudios Avanzados de Comunicación EEES" mientras que en la Pompeu Fabra ofrece el "Doctorado en Comunicación Social", teniendo quince líneas de investigación, siendo una de ellas "Comunicación e Interculturalidad".

i) La Facultad de la Universidad CEU-San Pablo continúa ofreciendo un título generalista denominado "Análisis de la Sociedad de la Información y nuevas formas de comunicación" sin concretar las líneas incluidas en dicho Doctorado.

j) La Facultad de Comunicación de la Universidad de Navarra oferta ocho líneas de investigación, sin que ninguna se adecúe a las líneas de investigación objeto de estudio.

k) El resto de la Facultades que configuran la muestra no aluden en sus páginas web al programa de investigación de los doctorados ofrecidos, reflejando únicamente los másteres. 
Realizado el análisis en la oferta de posgrados verificados, consideramos pertinente indicar aquellos programas que, aun no siendo oficiales y siendo titulaciones propias, conviven y casan con las líneas de investigación propuestas y que surgen de la propia investigación.

La Facultad de Comunicación de laUniversidad Autónoma de Barcelona (AUB) ofrece dos máster y tres cursos de experto relacionados con la Cooperación Internacional en el marco de la comunicación y una asignatura de Responsabilidad Social en el Máster de Relaciones Públicas y Gabinetes de Comunicación denominada "Patrocinio, Mecenazgo y Responsabilidad Social Corporativa".

La Facultad de Ciencias Sociales y de la Comunicación de la Universidad del País Vasco contempla un título propio que ofrece un módulo de Educación y Comunicación para la Paz y el Desarrollo (con un valor de 5 créditos) en el Máster en Cooperación Internacional Descentralizada: Paz y Desarrollo, que se ofrece como título propio.

Por su parte, la Facultad de Comunicación de la Universidad Pontificia de Salamanca oferta el Máster en Comunicación, Desarrollo y Cambio Social y Máster en Comunicación corporativa y planificación estratégica, que acoge un módulo de Responsabilidad Social Corporativa. Añadir que dicho centro también contempla un curso de experto en "Comunicación para el cambio social a través de la radio".

Es preciso apuntar que esta investigación ha sido realizada cuando todavía diversos másteres se encontraban en proceso de verificación, por lo que únicamente se ha contado como objeto de estudio aquellos que se poseían el estatus de oficiales, por lo que los resultados podrían verse alterados en los próximos meses.

\section{CONCLUSIONES}

El estudio presentado denota una presencia simbólica en la oferta de posgrados de Comunicación en los ámbitos de la Cooperación Internacional para el desarrollo y la Responsabilidad Social en aquellos programas impulsados en el contexto del EEES, teniendo por tanto una debilitada y nula representatividad.

$\mathrm{Su}$ presencia en los posgrados de la disciplina de la Comunicación se encuentra limitada a asignaturas testimoniales insertada en los programas, especialmente másteres, por lo que se aprecia que aún no se han visto fortalecidos dichos campos en las planificaciones oficiales verificados por la ANECA. 
Sin embargo, en las titulaciones propias, especialmente en el campo de la CID, encuentra una mayor significancia encontrándose programas específicos liderados por la Universidad Autónoma de Barcelona que acumula la mayor oferta de propuestas, marcando un sello de identidad y una puesta en valor de su escenificación académica. Además, se advierte en los posgrados una línea continuista en la vertiente academicista de las docencias impartidas, sin reforzarse la vertiente profesional.

El panorama expuesto nos desvela que se ha obviado casi completamente la recomendación que recoge el RD 1393/2007 de la oportunidad que se brinda para introducir materias y programas que apuestan por incentivar el compromiso social del alumnado, pues la escasez de las mismas brilla por su ausencia. Esta línea, casa con una reciente investigación sobre la oferta académica de grado "Estudios de comunicación en el Espacio Europeo de Educación Superior (EEES): Nuevos contenidos, nuevos perfiles profesionales", en la que se denunciaba las mismas circunstancia, el lamentable desaprovecho del marco que brindaba el EEES en un intento de lograr unos/unas egresadas con un bagaje cultural, político y social que fomente los valores universales como la solidaridad, la justicia, el respeto a la diferencia y al medio ambiente (Olmedo, Álvarez, Ruiz, 2010).

Por todo ello, y dado la ventana que nos ha ofrecido el EEES es necesario e ineludible abogar y apostar:

a) Por una mayor presencia de contenidos de carácter social, y en concreto, aquellos que aborden el ámbito de la Cooperación Internacional para el desarrollo y la Responsabilidad Social, como ejes que nutran a nuestros/as estudiantes de unos principios universales que deben estar presentes en las aulas y ser desgranados, cuestionados y debatidos como parte del proceso de aprendizaje y la adquisición de competencias en una realidad social cada vez más compleja.

b) Las instituciones académicas deben ser conscientes de que la introducción de dichos contenidos no es una mera cuestión de valentía o de seguir tendencias, sino de vislumbrar nuevos derroteros, en los cuales los actores sociales van requiriéndolo dado el dinamismo del actual entorno social. En este dinamismo, muchas veces demasiado veloz, el compromiso por lograr un mundo más igualitarios y digno para el conjunto de los seres humanos y respetuoso con el Planeta, es de todos y todas, y ninguna de las oportunidades pueden ser desestimadas. 


\section{REFERENCIAS}

Capacitarse. (2010). Qué es la Responsabilidad social según la ISO26000. Recuperado el 27 de junio de 2011, de http://iso26000.com.ar/2010/11/responsabilidad-socialconcepto-iso26000/.

Flores Mayorga, M. T., Roca Correa, D. \& Tena Parera, D. (2010). La congruencia profesional de las competencias específicas del grado en Publicidad y Relaciones Públicas de las universidades públicas de Cataluña. En Competencias y perfiles profesionales en los estudios de Ciencias de la Comunicación. Madrid: Fragua.

García Nieto, M. T., Viñarás Abad, M. (2010). Las Relaciones Públicas en el EEES. Nuevos retos nuevas necesidades. En Los estudios de Ciencias de Comunicación en el EEES. Madrid: Fragua.

Ministerio de Asuntos Exteriores y Cooperación. (2005). Plan Director de la Cooperación española 2005-2008. Recuperado el 12 de agosto de 2011, de http:/ / www.maec.es/SiteCollectionDocuments/Cooperaci\%C3\%B3n\%20espa\%C3 \%B1ola/Publicaciones/Plan_Director_Esp.pdf.

Ministerio de Educación. (2011). Datos y cifras del Sistema Universitario Español. Curso 2010/2011. Madrid: Secretaría General Técnica.

Olmedo Salar, S., Álvarez Novell, A.; Ruiz Mora, I. (2010). Estudios de comunicación en el Espacio Europeo de Educación Superior (EEES): Nuevos contenidos, nuevos perfiles profesionales. En Los estudios de Ciencias de la Comunicación en el EEES. Madrid: Fragua.

Ortiz Sobrino, M. (2009). La reconversión de la formación en comunicación desde el espacio europeo de educación superior. Icono 14, 14: 35-49.

Tünnermann Bernheim, C. (1998). El rol del docente en la educación superior del Siglo XXI . Recuperado el 27 de agosto de 2011, de http://ucyt.edu.ni/Download/EL_ROL_DEL_DOCENTE_EN_LA_E_S_DEL_SIGL O_XXI.pdf 


\section{Silvia Olmedo Salar}

Periodista, docente e investigadora en el Departamento de Periodismo de la Facultad de Ciencias de la Comunicación de la Universidad de Málaga. Su línea de investigación se ha centrado en el análisis de la aplicación de la comunicación en el ámbito de la Cooperación internacional y el desarrollo, la democratización mediática y la construcción de nuevos imaginarios desde la comunicación, la incidencia de las Tecnologías de la Información y de la Comunicación (TIC) y los modelos de comunicación participativa en los medios alternativos. Investigadora en el Proyecto de Excelencia de la Junta de Andalucía TIC 6593 y del Proyecto del Ministerio de Ciencia e Innovación CSO2011-29195.

\section{Isabel Ruiz Mora}

Relaciones públicas, investigadora y docente en el Departamento de Comunicación Audiovisual y Publicidad de la Universidad de Málaga. Desarrolla su actividad investigadora en el ámbito de la comunicación organizacional, más concretamente en la relación que guarda las Relaciones Públicas con la Responsabilidad Social. Su trabajo también aborda otras temáticas como el estado de los estudios de comunicación. Actualmente es coeditora de la Revista Internacional de Relaciones Pública y miembro de diferentes asociaciones como Asociación de Investigación en Relaciones Públicas (AIRRPP), Instituto de Investigación en Relaciones Públicas (IIRP), Asociación de Investigación en Comunicación (AEIC) y de European Communication Research and Education Association (ECREA 